\title{
Qubit-environment Negativity versus Fidelity of conditional environmental states for an NV-center spin qubit interacting with a nuclear environment
}

\author{
Małgorzata Strzałka \\ Department of Theoretical Physics, Wrockaw University of Technology, 50-370 Wrockaw, Poland \\ Damian Kwiatkowski \\ Institute of Physics, Polish Academy of Sciences, 02-668 Warsaw, Poland \\ Łukasz Cywiński \\ Institute of Physics, Polish Academy of Sciences, 02-668 Warsaw, Poland \\ Katarzyna Roszak \\ Department of Theoretical Physics, Wroctaw University of Technology, 50-370 Wroctaw, Poland
}

(Dated: November 21, 2019)

\begin{abstract}
We study the evolution of qubit-environment entanglement, quantified using Negativity, for NVcenter spin qubits interacting with an environment of ${ }^{13} \mathrm{C}$ isotope partially polarized nuclear spins in the diamond lattice. We compare it with the evolution of the Fidelity of environmental states conditional on the pointer states of the qubit, which can serve as a tool to distinguish between entangling and non-entangling decoherence in pure-dephasing scenarios. The two quantities show remarkable agreement during the evolution in a wide range of system parameters, leading to the conclusion that the amount of entanglement generated between the qubit and the environment is proportional to the trace that the joint evolution leaves in the environment.
\end{abstract}

\section{INTRODUCTION}

The study of system-environment or even qubitenvironment entanglement is seriously limited due to large sizes of the studied environments, which translates into few entanglement measures being available on the level of density matrix considerations. This would not be a problem if the joint system-environment state would be pure, but in most realistic scenarios the initial state of the environment is far from pure except for extremely low temperatures. In fact, the only measure which can serve to quantify entanglement between two systems of any size which can be calculated directly from the joint density matrix is Negativity [1, 2] (or closely related logarithmic Negativity [3]), which nevertheless requires diagonalization of a matrix of the same size as the joint systems' Hilbert space. Negativity has its limitations, since there exist entangled states which are not detected by it [4, [5], but it is the best available tool if the two potentially entangled systems are larger than a qubit and a qutrit and the purity of the system is less than one. All other measures require some form of minimization over possible representations of the states in different bases, which becomes highly cumbersome with growing system size [6-10].

Recently, relatively straightforward methods for detecting system-environment [11] and qubit-environment [12 entanglement have been found for a scenario limiting the type of Hamiltonians that drive the systemenvironment interaction. The type of Hamiltonians under study leads to pure dephasing of the system/qubit when the environment is traced out (it cannot cause transitions between a set of system pointer states [13, 14] which is singled out by the interaction itself). This may seem like a large loss of generality, but said type of system-environment interactions are abundant, especially in solid state scenarios [15-29], but also for trapped ions 30, 31.

The problem of the method is that it does not quantify the amount of entanglement, instead answering the question if system-environment entanglement is present at a given time after initialization of the system/qubit in a pure state. As there are no limitations on the initial state of the environment, which is likely to be mixed, the whole system is initially impure, and pure dephasing can occur either while being accompanied by entanglement generation or due to completely separable system-environemnt evolutions [11, 12, 32 34. This is in stark contrast to pure state system-environment evolutions, for which pure dephasing is irrefutably linked with the buildup of entanglement with the environment [14, 35]. The results of Refs [11, 12] show that system-environment entanglement leaves a detectable trace on the environment, while it is impossible to determine entangling from nonentangling evolutions by straightforward measurements of system pure dephasing. More involved schemes for the detection of qubit-environment entanglement by operations and measurements on only the qubit subsystem have been recently proposed [36]. Both, detection of entanglement by measurement only on the environment and detection of entanglement via operations on the qubit are possible because the problem is restricted to a special class of Hamiltonians, hence there is no contradiction with the popular theorem on the impossibility of local detection of entanglement.

The evolution of a qubit and its environment is not 
accompanied by entanglement generation if and only if the evolution of the states of the environment conditional on the pointer states of the qubit is the same at all times (if this occurs only at isolated points of time, then there is no entanglement only at these times) [12,

$$
\hat{R}_{00}(t)=\hat{R}_{11}(t)
$$

where said conditional states are denoted by $\hat{R}_{i i}(t)$, with $i=0,1$, and they correspond to the state the environment would be in at time $t$ if the qubit were initialized in pointer state $|i\rangle$ at the initial time. Hence if the qubit is initialized in a superposition state $a|0\rangle+b|1\rangle$, the state of the environment at time $t$, obtained by tracing out the qubit from the full qubit-environment density matrix, is given by

$$
\hat{R}(t)=|a|^{2} \hat{R}_{00}(t)+|b|^{2} \hat{R}_{11}(t) .
$$

In case there is no qubit-environment entanglement, this state is the same regardless on the initial qubit superposition and is equal to the state the environment would evolve to under the influence of the qubit in one of its pointer states. When there is entanglement generated with the environment, the situation is qualitatively different, and the state of the environment depends on the probability of finding the qubit in either pointer state. Therefore we can talk about a trace left by joint qubit-environment evolution on the environment which is present only for entangling evolutions.

Here, we make a first step towards a measure of qubit-environment entanglement, designed to quantify the amount of entanglement generating during evolutions of pure dephasing type. To this end, we test if the magnitude of the trace left by entangling evolutions on the state of the environment is proportional to the amount of actual entanglement generated on a realistically modeled NV-center in diamond spin qubit interacting with a nuclear spin environment [29]. The choice of test system is based both on its experimental relevance 37-42 and on the wide variety of test scenarios it gives. As the NVcenter has effectively spin $S=1$, the spin states form a qutrit, but the uneven level spacing between the different spin states allows for any two levels out of three to be singled out as the qubit under study. Furthermore, this type of spin qubits interact strongly only with nuclei of spinful carbon isotopes ${ }^{13} \mathrm{C}$, which are few within the diamond crystal lattice, and both their number and locations vary, which leads to different evolutions. The whole qubit-environment Hilbert space is therefore small enough to allow for effective diagonalization of matrices within it, which is necessary to find the evolution of Negativity.

We test a number of qubit-environment evolutions driven by different interaction Hamiltonians, all within the NV-center spin qubit model with five relevant environment nuclei. We find a remarkable agreement between the time-evolution of the entanglement measure Negativity and the Fidelity between the states of the environment conditional on the qubit pointer states (the difference between the two conditional environmental states serves to test for qubit-environment entanglement in Ref. [12]). Furthermore we find that this agreement is also present for evolutions which cannot be detected by the qubitbased scheme of Ref. [36. We conjecture that the effect is of more general nature and that said Fidelity could be the basis of an entanglement measure designed specifically for pure dephasing evolutions.

The paper is organized as follows. We introduce the $\mathrm{NV}$ center qubit and its environment in Sec. III In Sec. III we provide the definitions necessary to calculate qubitenvironment Negativity. In Sec. [V] we discuss the correlation between entanglement generation in pure dephasing scenarios and the difference between conditional evolution of the environment and use the Fidelity to quantify the difference. Results obtained for realistically modeled spin qubits with randomly chosen environments are presented and discussed in Sec. V] while Sec. VI contains concluding remarks.

\section{NV CENTER INTERACTING WITH A PARTIALLY POLARIZED NUCLEAR ENVIRONMENT}

Our test system consists of a spin qubit defined on an $\mathrm{NV}$ center in diamond interacting with an environment of nuclear spins of the spinful carbon isotope, ${ }^{13} \mathrm{C}$. As most of the diamond crystal lattice consists of spinless carbon nuclei, the relevant atoms of the environment (for decoherence) are few and randomly located. This is of use for testing of the correlation between generated entanglement and the magnitude of the trace that entangling evolutions leave on the conditional states of the environment (how strongly the conditional states of the environment are affected by entangling evolution), since the resulting system-environment evolutions vary depending on the choice of qubit as well as depending on the locations of the relevant carbon isotope atoms and their number.

The low energy states of the center constitute an effective electronic spin $S=1$, so we are dealing with a qutrit defined on the $m=-1,0$ and 1 levels, subsequently labeled as $|-1\rangle,|0\rangle$ and $|1\rangle$. This is subjected to a zero-field splitting $\Delta\left(S^{z}\right)^{2}$, with the direction of $z$ axis determined by the geometry of the center, so the presence of a magnetic field along the $z$ axis leads to a splitting of the $m_{s}= \pm 1$ levels and an uneven level spacing between them. This allows for any two-level subspace to be used as a qubit controlled by microwave electromagnetic fields. We choose two out of the three possible qubits for our study, one is the most widely employed qubit based on the $m=0$ and 1 levels, and the other is based on the $m=-1$ and 1 levels.

The large value of the zero-field splitting, $\Delta=2.87$ $\mathrm{GHz}$ and a large ratio of electronic and nuclear gyromagnetic factors lead to the suppression of transitions 
between the qutrit states mediated by the environment, hence the system can be described as one which undergoes only pure dephasing type of interaction [43]. Additionally, the $|0\rangle$ state is decoupled from the environment, so the qutrit-environment Hamiltonian is of the form

$$
\begin{aligned}
\hat{H}= & \left(\Delta+\gamma_{e} B_{z}\right)|-1\rangle\left\langle-1\left|+\left(\Delta-\gamma_{e} B_{z}\right)\right| 1\right\rangle\langle 1|+\hat{H}_{E} \\
& -|-1\rangle\langle-1|\otimes \hat{V}+| 1\rangle\langle 1| \otimes \hat{V} .
\end{aligned}
$$

The first two terms in the Hamiltonian describe the free evolution of the qutrit. The energy of states $| \pm 1\rangle$ depend on the zero-field splitting symmetrically and asymmetrically on a magnetic-field-dependent term, where $\gamma_{e}=28.08 \mathrm{MHz} / \mathrm{T}$ is the electron gyromagnetic ratio. This part of the Hamiltonian commutes with all other terms in eq. (3) and the resulting evolution can therefore be eliminated from the joint system-environment evolution via a unitary operation performed solely on the qutrit (by moving to a rotation frame with respect to the qubit). The consequence of this is that it has no bearing on either the generation of entanglement or on its magnitude. It will also play no part in the conditional evolution of the environment.

The second term in the Hamiltonian describes the free evolution of environmental spins,

$$
\hat{H}_{E}=\sum_{k} \gamma_{n} B_{z} \hat{I}_{k}^{z}
$$

where $k$ labels the spins, $\gamma_{n}=10.71 \mathrm{MHz} / \mathrm{T}$ is the gyromagnetic ratio for ${ }^{13} \mathrm{C}$ nuclei, $\hat{I}_{k}^{z}$ is the operator of the $z$ component of nuclear spin $k$. A term describing the internuclear magnetic dipolar interactions has been omitted, since the free evolution decoherence process occurs on much shorter timescales than said interactions (in contrast to coherence observed in spin echo experiment [29, 43]).

The last term in eq. (3) describes the hyperfine interaction between the spin qubit and its nuclear spin environment. It is given by

$$
\hat{V}=\sum_{k} \sum_{j \in(x, y, z)} \mathbb{A}_{k}^{z, j} \hat{I}_{k}^{j} .
$$

If we omit the Fermi contact interaction [44] which is related to the non-zero probability of finding an electron bound to the NV center on the location of a given nucleus, and only take the dipolar coupling into account, the coupling constants present in eq. (5) are given by

$$
\mathbb{A}_{k}^{z, j}=\frac{\mu_{0}}{4 \pi} \frac{\gamma_{e} \gamma_{n}}{r_{k}^{3}}\left(1-\frac{3\left(\mathbf{r}_{k} \cdot \hat{\mathbf{j}}\right)\left(\mathbf{r}_{k} \cdot \hat{\mathbf{z}}\right)}{r_{k}^{2}}\right) .
$$

Here, $\mu_{0}$ is the magnetic permeability of the vacuum, $\mathbf{r}_{k}$ is a displacement vector between the $k$-th nucleus and the qubit and $\hat{\mathbf{j}}=\hat{\mathbf{x}}, \hat{\mathbf{y}}, \hat{\mathbf{z}}$ denote versors corresponding to three distinct directions.

Note that the free evolution of the environment and the interaction term do not commute for non-zero magnetic fields, therefore the free evolution cannot be eliminated via a local unitary transformation and can take part in the generation of qubit-environment entanglement, regardless of the qubit of choice. Hence, the evolution operator for the qutrit and the environment (without the irrelevant free evolution of the qutrit) is given by

$$
\hat{U}(t)=\sum_{m=-1}^{1}|m\rangle\langle m| \otimes \hat{w}_{m}(t)
$$

with

$$
\begin{aligned}
\hat{w}_{-1}(t) & =e^{-\frac{i}{\hbar}\left(\hat{H}_{E}-\hat{V}\right) t} \\
\hat{w}_{0}(t) & =e^{-\frac{i}{\hbar} \hat{H}_{E} t} \\
\hat{w}_{1}(t) & =e^{-\frac{i}{\hbar}\left(\hat{H}_{E}+\hat{V}\right) t} .
\end{aligned}
$$

In the following we will be considering an initial state which is a product of a pure state of the qutrit within one of the two chosen qubit subspaces, $|\psi\rangle=a|0\rangle+b|1\rangle$ or $|\psi\rangle=a|-1\rangle+b|1\rangle$, and a partially polarized state of the nuclear environment, $\hat{R}(0)$, (which is mixed),

$$
\hat{\sigma}(0)=|\psi\rangle\langle\psi| \otimes \hat{R}(0) .
$$

The Hamiltonian (3) does not contain any terms which allow for transitions between different qutrit pointer states $|m\rangle$, so the effectively the evolution of such an initial state is governed only by the term in the Hamiltonian which contain the relevant qubit states, so either $|0\rangle$ and $|1\rangle$ or $|-1\rangle$ and $|1\rangle$. We assume that $\hat{R}(0)$ does not contain any correlations between the nuclei, so $\hat{R}(0)=\bigotimes_{k} \hat{\rho}_{k}$, where $\hat{\rho}_{k}$ is the density matrix of $k$-th nucleus, given in the case of spin- $1 / 2$ nuclei by

$$
\hat{\rho}_{k}=\frac{1}{2}\left(\mathbb{1}+2 p_{k} \hat{I}_{k}^{z}\right),
$$

where $p_{k} \in[-1,1]$ is the polarization of the $k$-th nucleus. Without dynamic nuclear polarization, $p_{k}=0$ for all $k$, the density operator of the environment at low fields is $\hat{R}(0) \propto \mathbb{1}$, and according to the results of Ref. [12] no qubit environment entanglement would form throughout the evolution. Since such nuclear polarization of the environment for an NV center has been recently mastered [45 [53], the assumption of the specially prepared initial state of the environment is reasonable. In the following we will assume that the polarizations of each environmental nucleus are the same, so $p_{k}=p$ for all $k$.

Since both the initial state and the evolution operator are known, we can write the time-evolved qubitenvironment density matrix in the form

$$
\tilde{\sigma}(t)=\left(\begin{array}{cc}
|a|^{2} \hat{R}_{n n}(t) & a b^{*} \hat{R}_{n 1}(t) \\
a^{*} b \hat{R}_{1 n}(t) & |b|^{2} \hat{R}_{11}(t)
\end{array}\right),
$$

with $n=-1,0$ depending on the choice of the qubit. Here the environmental operators $\hat{R}_{i j}(t)$ are given by

$$
\hat{R}_{i j}(t)=\hat{w}_{i}(t) \hat{R}(0) \hat{w}_{j}^{\dagger}(t) .
$$




\section{NEGATIVITY - AN ENTANGLEMENT MEASURE APPLICATIVE FOR LARGE BIPARTITE SYSTEMS}

For large bipartite systems, such as the studied here qubit and environment (where the latter is larger), the choice of entanglement measures which can be computed is very limited. It comes down in fact practically to the choice between Negavitity [1, 2] or logarithmic Negativity [3]. Both measures are closely related and are based on the positive partial transpose (PPT) criterion of separability [54, 55]. The criterion and therefore also the measures do not detect a certain type of entangled states called bound entangled states [4, 5], but in the studied scenario, namely in the case of an initially pure-state qubit, bound entanglement never forms [12, 56]. Therefore in what follows, Negativity (and logarithmic Negativity) signifies separability if and only if the joint qubit and environment state is really separable.

In what follows, we choose to employ plain Negativity. It is defined as the absolute sum of the negative eigenvalues of the density matrix of the whole system after a partial transposition with respect to one of the two potentially entangled subsystems and can be written as

$$
N(\hat{\sigma})=\sum_{i} \frac{\left|\lambda_{i}\right|-\lambda_{i}}{2}
$$

where $\lambda_{i}$ denote all eigenvalues of the density matrix after partial transposition, $\hat{\sigma}^{\Gamma_{A}}$. Obviously the positive eigenvalues cancel out in eq. 13. while only negative eigenvalues are left. Negativity does not depend on the system with respect to which partial transposition is performed, $A=Q, E$.

We calculate negativity at each instance of time by first performing partial transposition with respect to the qubit on the time-evolved qubit-environment density matrix 11,

$$
\tilde{\sigma}^{\Gamma_{Q}}(t)=\left(\begin{array}{cc}
|a|^{2} \hat{R}_{n n}(t) & a^{*} b \hat{R}_{1 n}(t) \\
a b^{*} \hat{R}_{n 1}(t) & |b|^{2} \hat{R}_{11}(t)
\end{array}\right),
$$

and then finding the eigevalues of the matrix obtained in this way.

\section{FIDELITY OF CONDITIONAL ENVIRONMENTAL STATES}

As shown in Refs 11, 12, the if and only if criterion of separability for pure-dephasing qubit-environment evolutions at time $t$ can be written as

$$
\hat{R}_{n n}(t)=\hat{R}_{11}(t)
$$

where the density matrices of the environment conditional on the qubit being in either of its pointer states is given by eq. 12 . This means that there is no entanglement between the qubit and the environment at time $t$,

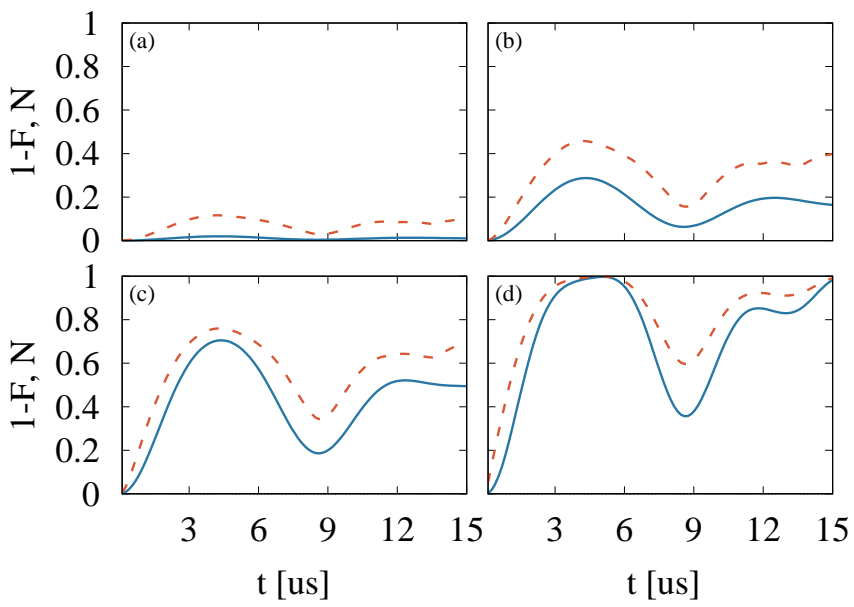

FIG. 1. Evolution of qubit-environment Negativity (red dashed lines) and one-minus-Fidelity between conditional environmental states (blue solid lines) for a qubit defined on $m=0$ and $m=1$ spin states and five environmental spins at random locations as a function of time for zero magnetic field and different initial polarizations of the environment: (a) $p=0.1$, (b) $p=0.4$, (c) $p=0.7$, (d) $p=1$.

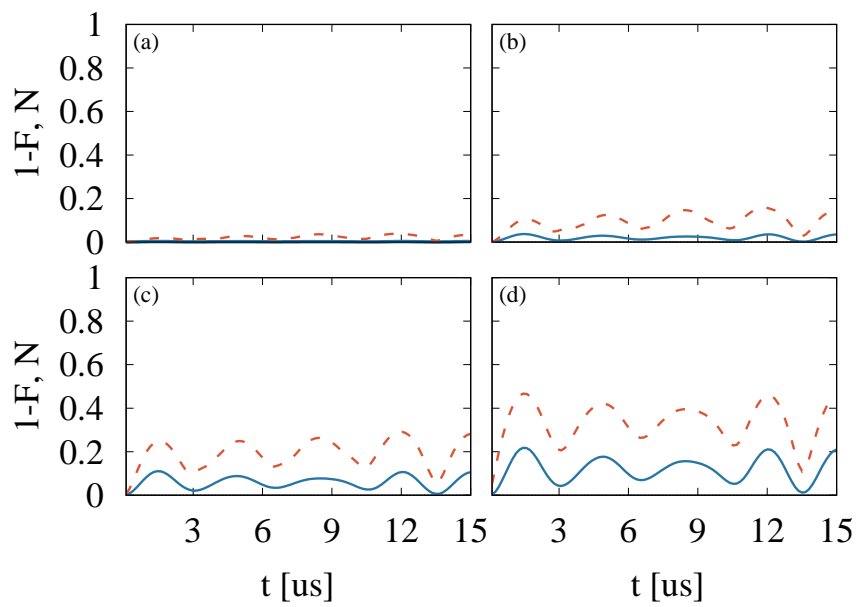

FIG. 2. Evolution of qubit-environment Negativity (red dashed lines) and one-minus-Fidelity between conditional environmental states (blue solid lines) for a qubit defined on $m=0$ and $m=1$ spin states and five environmental spins at random locations as a function of time for $B_{z}=0.2 \mathrm{~T}$ and different initial polarizations of the environment: (a) $p=0.1$, (b) $p=0.4$, (c) $p=0.7$, (d) $p=1$.

for an initial state that involves a pure state superposition in the qubit subspace, if and only if the environment would be in the same state at time $t$ if the qubit would have been initialized in either of its pointer states.

We conjecture that the degree of how different the two conditional density matrices are, is proportional to the amount of entanglement generated throughout the evo- 


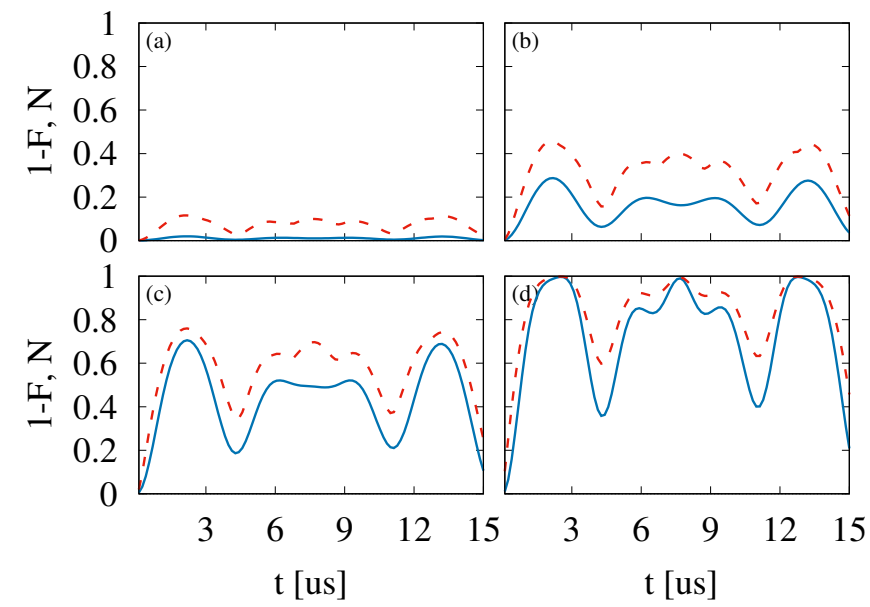

FIG. 3. Evolution of qubit-environment Negativity (red dashed lines) and one-minus-Fidelity between conditional environmental states (blue solid lines) for a qubit defined on $m=-1$ and $m=1$ spin states and five environmental spins at random locations as a function of time for zero magnetic field and different initial polarizations of the environment: (a) $p=0.1$, (b) $p=0.4$, (c) $p=0.7$, (d) $p=1$.

lution. To quantify this difference we will use the Fidelity between $\hat{R}_{n n}(t)$ and $\hat{R}_{11}(t)$, which yields a number between zero and one, one meaning that the states are the same and zero that they have orthogonal supports. The definition of Fidelity for two arbitrary density matrices (of the same dimensionality) $\hat{R}_{n n}$ and $\hat{R}_{11}$ is

$$
F\left(\hat{R}_{n n}, \hat{R}_{11}\right)=\left[\operatorname{Tr}\left(\sqrt{\sqrt{\hat{R}_{n n}} \hat{R}_{11} \sqrt{\hat{R}_{n n}}}\right)\right]^{2} .
$$

\section{RESULTS}

In the following we compare the evolution of Negativity between one of the two chosen qubits and the environment and one minus the Fidelity between the conditional states of the environment, $1-F\left(\hat{R}_{n n}(t), \hat{R}_{11}(t)\right)$, where $n=-1,0$ is specified by the choice of qubit. As the aim here is to study exemplary evolutions of the type as can be found in NV-center qubits interacting with a nuclear environment, we use the same randomly chosen realization of the spin environment in all plots. They correspond to an environment composed of five ${ }^{13} \mathrm{C}$ isotopes (nuclear spin $1 / 2$ ) for which their randomly generated spacial arrangement determines the coupling constants (6).

The evolution of Negativity between the $m=0,1$ qubit and an environment is plotted for $B_{z}=0$ and $B_{z}=0.2 \mathrm{~T}$ in Figs 1 and 2 , respectively, using dashed red lines. For the $m=-1,1$ qubit analogous plots are found in Fig. 3 for $B_{z}=0$ and in Fig. 4 for $B_{z}=0.2 \mathrm{~T}$. Analogously, the evolution of the one-minus-Fidelity between the conditional states of the environment, $1-F\left(\hat{R}_{n n}(t), \hat{R}_{11}(t)\right)$,

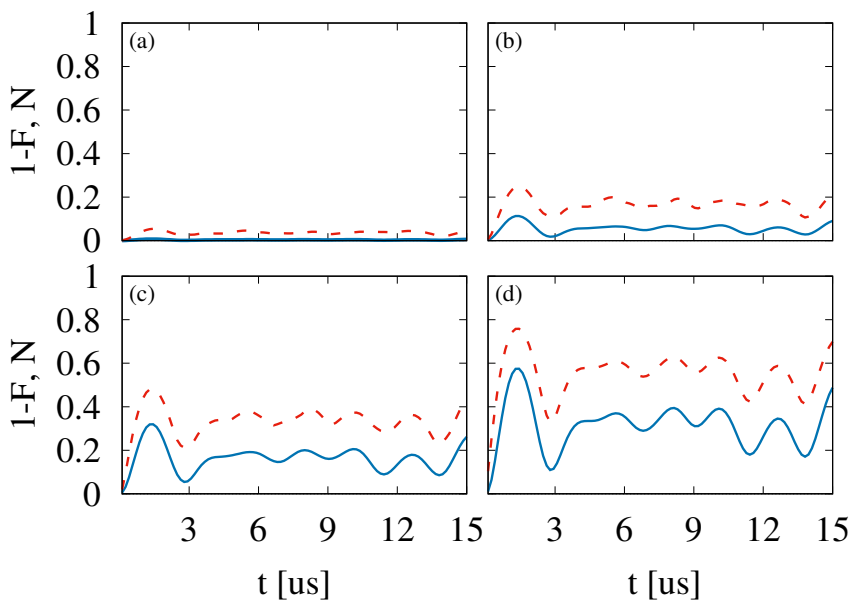

FIG. 4. Evolution of qubit-environment Negativity (red dashed lines) and one-minus-Fidelity between conditional environmental states (blue solid lines) for a qubit defined on $m=-1$ and $m=1$ spin states and five environmental spins at random locations as a function of time for $B_{z}=0.2 \mathrm{~T}$ and different initial polarizations of the environment: (a) $p=0.1$, (b) $p=0.4$, (c) $p=0.7$, (d) $p=1$.

is plotted in the same figures using solid blue lines, with $n=0$ for Figs 1 and 2 and with $n=-1$ for Figs 3 and 4 . The panels (a), (b), (c), and (d) in all plots correspond to growing initial polarization of the environment, with the most mixed environment (corresponding to $p=1$, so not maximally mixed) in panels (a) and fully polarized environments in panels (d). All of the figures contain results for an initial equal superposition qubit state (the initial phase between the components of this superposition is irrelevant).

As should be expected [12, for a completely mixed environment, $p=0$, qubit decoherence is not accompanied by the generation of entanglement regardless of the type of interaction with environment, since the initial density matrix of the environment is proportional to unity and commutes with any possible environmental evolution operators [12]. This does not mean that the qubit does not experience decoherence and, in fact, the qubit becomes dephased during the evolution in all four of the studied situations with not polarized initial states of the environment.

For partially and fully polarized initial environmental states, generation of entanglement is observed regardless of the variant of the Hamiltonian under study. This has been predicted for the $m=0,1$ qubit when $B_{z} \neq 0$, which has been used to exemplify the scheme for detection of qubit-environment entanglement via operations only on the qubit subsystem [36. The procedure described there could also be used to predict the generation of qubitenvironment entanglement for the $m=-1,1$ qubit and $B_{z} \neq 0$. This is because the condition for the procedure described in Ref. [36] to be able to detect qubit- 
environment entanglement is for the evolution operators on the environment conditional on the pointer state of the qubit (8) not to commute, so

$$
\left[\hat{w}_{n}(t), \hat{w}_{1}(t)\right] \neq 0,
$$

with $n=-1,0$ depending on the choice of qubit. This condition is met for $B_{z} \neq 0$, but not for $B_{z}=0$ when $\hat{w}_{-1}(t)=\hat{w}_{1}^{\dagger}(t)$ and $\hat{w}_{0}(t)=\mathbb{I}$.

More interestingly, the evolution of the quantity $1-$ $F\left(\hat{R}_{n n}(t), \hat{R}_{11}(t)\right)$ which determines how different the two conditional states of the environment are at time $t$, resembles the evolution of the Negativity very closely. In fact, $1-F\left(\hat{R}_{n n}(t), \hat{R}_{11}(t)\right)$ grows when Negativity grows, decreases when Negativity decreases, and remains constant when Negativity remains constant. To exemplify this, we plot the time-derivatives of both Negativity and one-minus-Fidelity corresponding to the evolutions in Fig. 1 in Fig. 5. It can be seen that the derivatives of both quantities are positive, negative, and equal to zero at the same segments or points of time.

Since this is the case in all four situations studied, which although they correspond to one physical scenario differ quite extensively, containing an asymmetric system-environment coupling (the $m=0,1$ qubit) with $\left(B_{z}=0\right)$ and without $\left(B_{z} \neq 0\right)$ commuting environmental and interaction parts of the Hamiltonian, as well as a coupling which is not asymmetric (the $m=-1,1$ qubit) again in two variants as pertains the commutation of parts of the Hamiltonian, it is reasonable to assume that the close resemblance of the Negativity and one-minus-Fidelity evolutions is not accidental.

\section{CONCLUSION}

We have studied four variations of an NV-center spin qubit interacting with an environment of a few nuclear spins, which in all cases leads to pure dephasing of the qubit. The variations are obtained by the choice of qubit under study (we chose two out of three possible qubits) which yields different effective interaction Hamiltonians and by the application of the magnetic field or lack thereof. The latter facilitates the transition between commuting and non-commuting conditional evolution operators of the environment and is important from the point of view of detecting this type of entanglement.

We have compared the time-evolution of the amount of entanglement between the qubit and the environment with the time-evolution of one minus the Fidelity of the state of the environment at time $t$ on the qubit pointer state. In all studied situations the evolution of oneminus-Fidelity resembled the evolution of Negativity very closely, to the extent that both quantities were growing and decreasing in the same time-segments.
We conjecture that the amount of entanglement with the environment generated during any evolution that leads to pure dephasing of the qubit for an initial product state of a pure qubit and environment is propor-

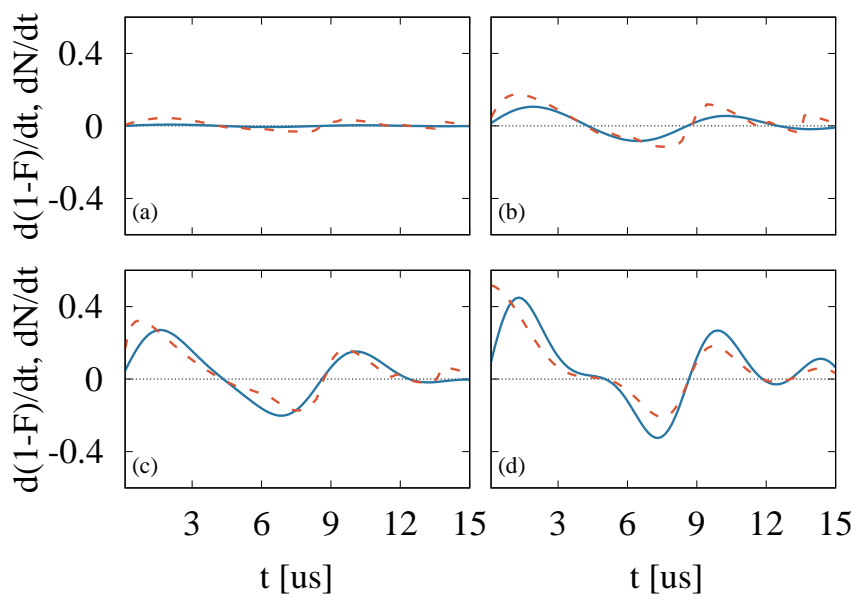

FIG. 5. Time-derivative of the evolution of qubit-environment Negativity (red dashed lines) and one-minus-Fidelity between conditional environmental states (blue solid lines) for a qubit defined on $m=0$ and $m=1$ spin states and five environmental spins at random locations as a function of time for zero magnetic field and different initial polarizations of the environment: (a) $p=0.1$, (b) $p=0.4$, (c) $p=0.7$, (d) $p=1$. The figure corresponds to the evolutions in Fig. (1).

tional to one-minus-Fidelity between the states of the environment conditional on the qubit pointer states. This would mean that the amount of entanglement generated between the qubit and the environment is proportional to the trace that the joint evolution leaves on the environment. Hence, although it is not possible to distinguish between entangling and nonentangling evolutions by studying the level of qubit dephasing, it is not only possible to distinguish them by detecting the difference in environmental evolution linked to the different pointer states of the qubit, but it may also be possible to quantify the amount of entanglement in the qubit-environment system by studying the magnitude of this difference. We have shown this to be the case in quantitatively different situations which can be realized in NV-center spin qubits. The advantage is that contrary to other measures of mixed-state entanglement, here we have a natural physical interpretation, which in fact is the same as for the pure state entanglement in pure dephasing scenarios (namely, how much the two conditional states of the environment differ from one another).

This work was supported by funds of the Polish National Science Center (NCN), Grant no. 2015/19/B/ST3/03152. 
[1] G. Vidal and R. F. Werner, "Computable measure of entanglement," Phys. Rev. A 65, 032314 (2002).

[2] J. Lee, M.S. Kim, Y.J. Park, and S. Lee, "Partial teleportation of entanglement in a noisy environment," J. Mod. Opt. 47, 2157 (2000).

[3] M. B. Plenio, "Logarithmic negativity: A full entanglement monotone that is not convex," Phys. Rev. Lett. 95, 090503 (2005)

[4] Paweł Horodecki, "Separability criterion and inseparable mixed states with positive partial transposition," Phys. Lett. A 232, 333-339 (1997).

[5] Michał Horodecki, Paweł Horodecki, and Ryszard Horodecki, "Mixed-state entanglement and distillation: Is there a "bound" entanglement in nature?" Phys. Rev. Lett. 80, 5239-5242 (1998)

[6] F. Mintert, A. R. R. Carvalho, M. Kuś, and A. Buchleitner, "Measures and dynamics of entangled states," Phys. Rep. 415, 207 (2005).

[7] Martin B. Plenio and Shashank Virmani, "An introduction to entanglement measures," Quant. Info. Comput. 7, 1 (2007).

[8] Ryszard Horodecki, Paweł Horodecki, Michał Horodecki, and Karol Horodecki, "Quantum entanglement," Rev. Mod. Phys. 81, 865-942 (2009)

[9] Leandro Aolita, Fernando de Melo, and Luiz Davidovich, "Open-system dynamics of entanglement," Rep. Prog. Phys. 78, 042001 (2015)

[10] B. Kraus, J. I. Cirac, S. Karnas, and M. Lewenstein, "Separability in $2 \times n$ composite quantum systems," Phys. Rev. A 61, 062302 (2000).

[11] Katarzyna Roszak, "Criteria for system-environment entanglement generation for systems of any size in puredephasing evolutions," Phys. Rev. A 98, 052344 (2018).

[12] Katarzyna Roszak and Łukasz Cywiński, "Characterization and measurement of qubit-environmententanglement generation during pure dephasing," Phys. Rev. A 92, 032310 (2015).

[13] W. H. Zurek, "Pointer basis of quantum apparatus: Into what mixture does the wave packet collapse?"'Phys. Rev. D 24, 1516-1525 (1981)

[14] Wojciech Hubert Zurek, "Decoherence, einselection, and the quantum origins of the classical," Rev. Mod. Phys. 75, 715 (2003)

[15] J. Medford, Ł. Cywiński, C. Barthel, C. M. Marcus, M. P. Hanson, and A. C. Gossard, "Scaling of dynamical decoupling for spin qubits," Phys. Rev. Lett. 108, 086802 (2012)

[16] Erika Kawakami, Thibaut Jullien, Pasquale Scarlino, Daniel R. Ward, Donald E. Savage, Max G. Lagally, Viatcheslav V. Dobrovitski, Mark Friesen, Susan N. Coppersmith, Mark A. Eriksson, and Lieven M. K. Vandersypen, "Gate fidelity and coherence of an electron spin in an si/sige quantum dot with micromagnet," PNAS 113, $11738(2016)$

[17] P. Borri, W. Langbein, S. Schneider, U. Woggon, R. L. Sellin, D. Ouyang, and D. Bimberg, "Ultralong dephasing time in InGaAs quantum dots," 87, 157401-1-4 (2001).

[18] A. Vagov, V. M. Axt, T. Kuhn, W. Langbein, P. Borri, and U. Woggon, "Nonmonotonous temperature dependence of the initial decoherence in quantum dots," $\mathbf{7 0}$,
201305(R)-1-4 (2004).

[19] Katarzyna Roszak and Paweł Machnikowski, "Complete disentanglement by partial pure dephasing," Phys. Rev. A 73, 022313 (2006).

[20] E. Paladino, Y. M. Galperin, G. Falci, and B. L. Altshuler, " $1 / f$ noise: Implications for solid-state quantum information," Rev. Mod. Phys. 86, 361 (2014).

[21] P. Szańkowski, G. Ramon, J. Krzywda, D. Kwiatkowski, and Ł. Cywiński, "Environmental noise spectroscopy with qubits subjected to dynamical decoupling," J. Phys.:Condens. Matter 29, 333001 (2017).

[22] W. M. Witzel and S. Das Sarma, "Quantum theory for electron spin decoherence induced by nuclear spin dynamics in semiconductor quantum computer architectures: Spectral diffusion of localized electron spins in the nuclear solid-state environment," Phys. Rev. B 74, $035322(2006)$.

[23] Wang Yao, Ren-Bao Liu, and L. J. Sham, "Theory of electron spin decoherence by interacting nuclear spins in a quantum dot," Phys. Rev. B 74, 195301 (2006).

[24] Łukasz Cywiński, Wayne M. Witzel, and S. Das Sarma, "Pure quantum dephasing of a solid-state electron spin qubit in a large nuclear spin bath coupled by longrange hyperfine-mediated interaction," Phys. Rev. B 79, 245314 (2009)

[25] W. A. Coish, Jan Fischer, and Daniel Loss, "Freeinduction decay and envelope modulations in a narrowed nuclear spin bath," Phys. Rev. B 81, 165315 (2010).

[26] Hendrik Bluhm, Sandra Foletti, Izhar Neder, Mark Rudner, Diana Mahalu, Vladimir Umansky, and Amir Yacoby, "Long coherence of electron spins coupled to a nuclear spin bath," Nat. Phys. 7, 109 (2010).

[27] Wen Yang, Wen-Long Ma, and Ren-Bao Liu, "Quantum many-body theory for electron spin decoherence in nanoscale nuclear spin baths," Rep. Prog. Phys. 80, 016001 (2017)

[28] Filip K. Malinowski, Frederico Martins, Peter D. Nissen, Edwin Barnes, Łukasz Cywiński, Mark S. Rudner, Saeed Fallahi, Geoffrey C. Gardner, Michael J. Manfra, Charles M. Marcus, and Ferdinand Kuemmeth, "Notch filtering the nuclear environment of a spin qubit," Nature Nanotechnology 12, 16 (2017).

[29] Damian Kwiatkowski and Łukasz Cywiński, "Decoherence of two entangled spin qubits coupled to an interacting sparse nuclear spin bath: Application to nitrogen vacancy centers," Phys. Rev. B 98, 155202 (2018)

[30] Michael J. Biercuk, Hermann Uys, Aaron P. VanDevender, Nobuyasu Shiga, Wayne M. Itano, and John J. Bollinger, "Optimized dynamical decoupling in a model quantum memory," Nature 458, 996 (2009)

[31] Thomas Monz, Philipp Schindler, Julio T. Barreiro, Michael Chwalla, Daniel Nigg, William A. Coish, Maximilian Harlander, Wolfgang Hänsel, Markus Hennrich, and Rainer Blatt, "14-qubit entanglement: Creation and coherence," Phys. Rev. Lett. 106, 130506 (2011).

[32] Jens Eisert and Martin B. Plenio, "Quantum and classical correlations in quantum brownian motion," Phys. Rev. Lett. 89, 137902 (2002)

[33] Stefanie Hilt and Eric Lutz, "System-bath entanglement in quantum thermodynamics," Phys. Rev. A 79, 010101 (2009) 
[34] A. Pernice and Walter T. Strunz, "Decoherence and the nature of system-environment correlations," Phys. Rev. A 84, 062121 (2011)

[35] Klaus Hornberger, "Introduction to decoherence theory," Lect. Notes Phys. 768, 221 (2009)

[36] Katarzyna Roszak, Damian Kwiatkowski, and Łukasz Cywiński, "How to detect qubit-environment entanglement generated during qubit dephasing," Phys. Rev. A 100, 022318 (2019)

[37] T. Staudacher, F. Shi, S. Pezzagna, J. Meijer, J. Du, C. A. Meriles, F. Reinhard, and J. Wrachtrup, "Nuclear magnetic resonance spectroscopy on a $(5 \text {-nanometer })^{3}$ sample volume," Science 339, 561 (2013)

[38] Stephen J. DeVience, Linh M. Pham, Igor Lovchinsky, Alexander O. Sushkov, Nir Bar-Gill, Chinmay Belthangady, Francesco Casola, Madeleine Corbett, Huiliang Zhang, Mikhail Lukin, Hongkun Park, Amir Yacoby, and Ronald L. Walsworth, "Nanoscale nmr spectroscopy and imaging of multiple nuclear species," Nature Nanotechnology 10, 129 (2015).

[39] T. Häberle, D. Schmid-Lorch, F. Reinhard, and J. Wrachtrup, "Nanoscale nuclear magnetic imaging with chemical contrast," Nature Nanotechnology 10, 125 (2015)

[40] I. Lovchinsky, A. O. Sushkov, E. Urbach, N. P. de Leon, S. Choi, K. De Greve, R. Evans, R. Gertner, E. Bersin, C. Müller, L. McGuinness, F. Jelezko, R. L. Walsworth, H. Park, and M. D. Lukin, "Nuclear magnetic resonance detection and spectroscopy of single proteins using quantum logic," Science 351, 836 (2016).

[41] J. Wrachtrup and A. Finkler, "Single spin magnetic resonance," J. Magn. Res. 369, 225 (2016).

[42] C. L. Degen, F. Reinhard, and P. Cappellaro, "Quantum sensing," Rev. Mod. Phys. 89, 035002 (2017).

[43] Nan Zhao, Sai-Wah Ho, and Ren-Bao Liu, "Decoherence and dynamical decoupling control of nitrogen vacancy center electron spins in nuclear spin baths," Phys. Rev. B 85, 115303 (2012).

[44] Adam Gali, Maria Fyta, and Efthimios Kaxiras, "Ab initio supercell calculations on nitrogen-vacancy center in diamond: Electronic structure and hyperfine tensors," Phys. Rev. B 77, 155206 (2008).

[45] P. London, J. Scheuer, J. M. Cai, I. Schwarz, A. Retzker, M. B. Plenio, M. Katagiri, T. Teraji, S. Koizumi, J. Isoya, R. Fischer, L. P. McGuinness, B. Naydenov, and F. Jelezko, "Detecting and polarizing nuclear spins with double resonance on a single electron spin," Phys. Rev. Lett. 111, 067601 (2013), arXiv:1304.4709.

[46] Ran Fischer, Christian O. Bretschneider, Paz London, Dmitry Budker, David Gershoni, and Lucio Frydman,
"Bulk nuclear polarization enhanced at room temperature by optical pumping," Physical Review Letters 111, 057601 (2013), arXiv arXiv:1211.5801 v1

[47] Daniela Pagliero, K. R. Koteswara Rao, Pablo R. Zangara, Siddharth Dhomkar, Henry H. Wong, Andrea Abril, Nabeel Aslam, Anna Parker, Jonathan King, Claudia E. Avalos, Ashok Ajoy, Joerg Wrachtrup, Alexander Pines, and Carlos A. Meriles, "Multispin-assisted optical pumping of bulk ${ }^{13} \mathrm{C}$ nuclear spin polarization in diamond," Phys. Rev. B 97, 024422 (2018).

[48] Ralf Wunderlich, Jonas Kohlrautz, Bernd Abel, Jürgen Haase, and Jan Meijer, "Optically induced cross relaxation via nitrogen-related defects for bulk diamond $\mathrm{C}$ 13 hyperpolarization," Physical Review B 96, 220407(R) (2017), arXiv:1703.09243

[49] Gonzalo A. Alvarez, Christian O. Bretschneider, Ran Fischer, Paz London, Hisao Kanda, Shinobu Onoda, Junichi Isoya, David Gershoni, and Lucio Frydman, "Local and bulk 13C hyperpolarization in nitrogen-vacancycentred diamonds at variable fields and orientations," $\mathrm{Na}-$ ture Communications 6, 8456 (2015), arXiv:1412.8635.

[50] Jonathan P. King, Keunhong Jeong, Christophoros C. Vassiliou, Chang S. Shin, Ralph H. Page, Claudia E. Avalos, Hai-Jing Wang, and Alexander Pines, "Room-temperature in situ nuclear spin hyperpolarization from optically pumped nitrogen vacancy centres in diamond," Nature Communications 6, 8965 (2015), arXiv:1501.2897

[51] Jochen Scheuer, Ilai Schwartz, Samuel Müller, Qiong Chen, Ish Dhand, Martin B. Plenio, Boris Naydenov, and Fedor Jelezko, "Robust techniques for polarization and detection of nuclear spin ensembles," Physical Review B 96, 174436 (2017), arXiv:1706.01315.

[52] F. Poggiali, P. Cappellaro, and N. Fabbri, "Measurement of the excited-state transverse hyperfine coupling in NV centers via dynamic nuclear polarization," Physical Review B 95, 195308 (2017), arXiv:1612.04783.

[53] Y. Hovav, B. Naydenov, F. Jelezko, and N. Bar-Gill, "Low-field nuclear polarization using nitrogen vacancy centers in diamonds," Phys. Rev. Lett. 120, 060405 (2018)

[54] Asher Peres, "Separability criterion for density matrices," Phys. Rev. Lett. 77, 1413-1415 (1996).

[55] Michał Horodecki, Paweł Horodecki, and Ryszard Horodecki, "Separability of mixed states: necessary and sufficient conditions," Phys. Lett. A 223, 1-8 (1996).

[56] Paweł Horodecki, Maciej Lewenstein, Guifré Vidal, and Ignacio Cirac, "Operational criterion and constructive checks for the separability of low-rank density matrices," Phys. Rev. A 62, 032310 (2000). 